\title{
Classical Diffusion of a quantum particle in a noisy environment
}

\author{
Ariel Amir, Yoav Lahini and Hagai B. Perets \\ Faculty of Physics, Weizmann Institute of Science, Rehovot, 76100, Israel
}

\begin{abstract}
We study the spreading of a quantum-mechanical wavepacket in a one-dimensional tight-binding model with a noisy potential, and analyze the emergence of classical diffusion from the quantum dynamics due to decoherence. We consider a finite correlation time of the noisy environment, and treat the system by utilizing the separation of fast (dephasing) and slow (diffusion) processes. We show that classical diffusive behavior emerges at long times, and we calculate analytically the dependence of the classical diffusion coefficient on the noise magnitude and correlation time. This method provides a general solution to this problem for arbitrary conditions of the noisy environment. The results are relevant to a large variety of physical systems, from electronic transport in solid state physics, to light transmission in optical devices, diffusion of excitons, and quantum computation.
\end{abstract}

Introduction.- The dynamics of quantum particles, described by the Schrodinger equation, manifests rich and exciting physics. The dynamics of a quantum particle in homogenous or periodic media is ballistic - i.e., the meansquare displacement of a quantum particle grows linearly with time, in contrast to the diffusive propagation of a classical particle. In many realistic cases, such quantum particles are exposed to time-dependent noisy environments, and decoherence effects become important. In this work we focus on the emergence of seemingly classical diffusion from the quantum dynamics under such conditions. Specifically, we calculate the classical diffusion coefficient as a function of the parameters of the noise the magnitude and the correlation time. Our analytical calculations are found to be in excellent agreement with numerical simulations.

To formulate the transition from quantum to classical diffusion, we use a tight-binding model with a stochastic potential. The problem of the spreading of a quantum wave-packet in a dynamic, time-dependent potential has received much attention in the last three decades $[1,2,43,4,[5,6,6,6]$. More recently this problem was discussed in the context of quantum random walks with decoherence and quantum computation [9, 10]. This simplified theoretical problem is of great relevance to various experimental systems, such as particle diffusion in molecular crystals [2], diffusion of excitons 11], photon propagation in coupled waveguide lattices [12, 13], and destruction of Anderson localization by nonlinearity or dimensional crossover effects [14, 15, 16, 17]. The problem of dynamics and dephasing due to coupling to a thermal bath is also closely related [18, 19, 20]. Early works [1] showed that unlike the static disorder case, where Anderson localization sets in 21], diffusive behavior takes over at long enough times for a tight-binding model with timedependent disorder. Madhukar and Post [2] extended this to the case of on-site as well as off-diagonal disorder. Various works [3, 6, 22] dealt with the problem on a continuous lattice, which can show superdiffusive behavior. Heinrichs [5] showed the correspondence between discrete (tight-binding) and continuous scenarios. All of these works assumed, for theoretical simplicity, delta-function correlations in time (white noise). The corresponding experimental assumption for electron diffusion in molecular crystals, for example, is a temperature higher than the Debye temperature [2]. Clearly, one would like to extend the theoretical understanding to more realistic cases of finite correlation times of the noisy environment. A first step in that direction was undertaken by [23], using a perturbative analysis in the correlation time $\tau$. In this work we extend this to arbitrarily large correlation times. We use a novel method, relying on the separation of fast and slow processes (dephasing versus diffusion), to derive accurate analytical solutions for this problem.

Model and derivation.- We consider the Schrodinger equation for a one-dimensional tight-binding model, taking $\hbar=1$. The equations governing the process are:

$$
\left.i \frac{d A_{j}}{d t}=T\left(A_{j+1}+A_{j-1}\right)+\xi_{(j}, t\right) A_{j},
$$

where $A_{j}$ is the amplitude at site $j$ and $T$ is the nearestneighbor tunneling. The noise term $\xi(j, t)$ is assumed to be Gaussian and uncorrelated between different sites, and will be characterized by a time correlation function $C$ :

$$
\left\langle\xi\left(i, t^{\prime}\right) \xi\left(j, t^{\prime}+t\right)\right\rangle=\delta_{i j} C(t) .
$$

It is clear from the definition that $C(-t)=C(t)$. The typical time of the decay of $C(t)$ is defined as the correlation time $\tau$, and the typical magnitude is defined as $W$, i.e., $C(0)=W^{2}$. We shall assume that $T \ll W$, from which follows that the dynamics of the phases of $A_{j+1}$ and $A_{j-1}$ will be driven mainly by the second term of Eq. (11). In the lowest order approximation $A^{0}$ can be written as:

$$
A_{j}^{0}=\left|A_{j}^{0}\right| e^{-i \phi_{j}(t)},
$$

with $\phi_{j}(t)=\phi_{j}(0)+\int_{0}^{t} \xi\left(j, t^{\prime}\right) d t^{\prime}$.

Due to the random noise driving it, the phase $e^{-i \phi_{j}(t)}$ will also be a random variable, with a correlation function characterized by a correlation time (dephasing time) $\tau_{\phi} \neq$ $\tau$. We shall later show that this correlation function will be related to the diffusion constant of the particle. 
The problem of finding the correlation function, defined as $C_{\phi}\left(t-t^{\prime}\right) \equiv\left\langle e^{-i \phi_{j}(t)} e^{i \phi_{j}\left(t^{\prime}\right)}\right\rangle$, is equivalent to that encountered in the physics of nuclear magnetic resonance (NMR), where spins lose phase coherence due to the randomly fluctuating magnetic field created by the other spins. Plugging in the solution for $\phi_{j}(t)$, we obtain:

$$
C_{\phi}(\Delta t)=\left\langle e^{-i \int_{0}^{\Delta t} \xi(t) d t}\right\rangle,
$$

where we have omitted the site index from $\xi(j, t)$ since this calculation is essentially for an independent, arbitrary site.

Formally, calculating this average is similar to the determination of Debye-Waller factors 24] and yields:

$$
C_{\phi}(\Delta t)={ }_{\delta t \rightarrow 0}^{l i m} e^{-\frac{\delta t^{2}}{2}\left\langle\left(\sum_{k} \xi_{k}\right)^{2}\right\rangle}=e^{-\int_{0}^{\Delta t}(\Delta t-t) C(t) d t} .
$$

This is an exact result for the correlation function of the dephasing process. A similar result was obtained in [25], in the context of Dicke narrowing. When $\Delta t \gg \tau$, we can approximate the integral in the exponent of Eq. (5) by $\Delta t \int_{0}^{\infty} C(t)=\beta \Delta t W^{2} \tau$, where $\beta$ is non-universal and depends on the form of $C(t)$. For example, an exponential decay gives $\beta=1$, while a correlation function decaying linearly to zero gives $\beta=1 / 2$. Therefore in this regime:

$$
C_{\phi}(\Delta t) \approx e^{-\beta W^{2} \tau \Delta t}
$$

Thus for times large compared to $\tau$, the correlation time of the noise, the correlations of $e^{-i \phi}$ decay exponentially with a dephasing time $\tau_{\phi} \sim 1 / W^{2} \tau$. This is reminiscent of the well-known NMR phenomenon of motional narrowing [26].

For short times, $\Delta t \ll \tau$, we can approximate $C(t) \approx$ $C(0)=W^{2}$ in the integral of Eq. (5), and obtain:

$$
C_{\phi}(\Delta t) \approx e^{-\frac{W^{2}}{2} \Delta t^{2}} .
$$

Remarkably, in this limit the dephasing is insensitive to the form of the correlation function, and is given by a Gaussian with $\tau_{\phi}=\sqrt{2} / W$.

Let us now proceed to analyze the dynamics of the probability distribution of the particle, characterized by the set of probabilities $p_{j}=\left|A_{j}\right|^{2}$. It is clear that without the tunneling term $T$ the probabilities will remain constant, since the sites are uncoupled. However, due to the noise term, the phase of the amplitudes become a random variable, which, as we shall now show, will lead to classical diffusion of the probabilities.

From Eq. (11), one obtains the exact relation:

$$
\frac{d P_{j}}{d t}=2 T \operatorname{Im}\left[\mathrm{A}_{\mathrm{j}}^{*} \mathrm{~A}_{\mathrm{j}+1}+\mathrm{A}_{\mathrm{j}}^{*} \mathrm{~A}_{\mathrm{j}-1}\right] .
$$

Let us take an ensemble average of Eq. (8). To the zeroth order approximation, given by Eq. (3), the phase of $A_{j}, A_{j+1}$ and $A_{j-1}$ are independent. This will lead, upon taking the time-average, to the incorrect result $\left\langle\frac{d P_{j}}{d t}\right\rangle=0$. To proceed to the next order $A^{1}$ in $T / W$, we have to solve the following first-order, linear differential equation:

$$
i \frac{A_{j}^{1}}{d t}-\xi_{j} A_{j}^{1}=T\left(A_{j+1}^{0}+A_{j-1}^{0}\right) .
$$

Defining an integration factor $\mu_{j}=e^{i \int_{0}^{t} \xi_{j}\left(t^{\prime}\right) d t^{\prime}}=e^{i \phi_{j}(t)}$, this can be rewritten in the form:

$$
\frac{d\left[A_{j}^{1} \mu_{j}\right]}{d t}=\mu_{j} \frac{T}{i}\left(A_{j+1}^{0}+A_{j-1}^{0}\right) .
$$

Upon integration we obtain:

$$
A_{j}^{1}=A_{j}^{0}+\frac{T}{i \mu_{j}(t)} \int_{0}^{t} \mu_{j}\left(t^{\prime}\right)\left(A_{j+1}^{0}+A_{j-1}^{0}\right) d t^{\prime} .
$$

A similar equation holds for $A_{j+1}$ and $A_{j-1}$.

Plugging this into Eq. (8) and taking the ensembleaverage we obtain:

$$
\left\langle\frac{d P_{j}}{d t}\right\rangle \approx 2 T \operatorname{Im}\left[\left(\mathrm{A}_{\mathrm{j}}^{0}+\mathrm{A}_{\mathrm{j}}^{1}\right)^{*}\left(\mathrm{~A}_{\mathrm{j}+1}^{0}+\mathrm{A}_{\mathrm{j}+1}^{1}\right)\right]+\leftrightarrow,
$$

where $\leftrightarrow$ denotes identical terms upon the substitution $j+1->j-1$.

$A_{j}^{0}, A_{j+1}^{0}$ and $A_{j-1}^{0}$ are independent, thus, only three terms remain in the average. The first of these is:

$$
I=\left\langle 2 T^{2} \operatorname{Re}\left[-\left(\mathrm{A}_{\mathrm{j}}^{0}\right)^{*} \int_{0}^{\mathrm{t}} \frac{\mu_{\mathrm{j}+1}\left(\mathrm{t}^{\prime}\right)}{\mu_{\mathrm{j}+1}(\mathrm{t})} \mathrm{A}_{\mathrm{j}}^{0} \mathrm{dt}^{\prime}\right]\right\rangle,
$$

while the other two are of similar form, obtained upon changing $(j+1) \rightarrow j$ and $j \rightarrow j \pm 1$.

Let us evaluate $I$. Plugging in $A_{j}^{0}=P_{j}(t) e^{-i \phi_{j}(t)}$, and the explicit form of $\mu_{j+1}(t)$, we get:

$$
I=\left\langle P_{j}(t) \int_{0}^{t} e^{i\left[\phi_{j}(t)-\phi_{j}\left(t^{\prime}\right)\right]} e^{i\left[\phi_{j+1}(t)-\phi_{j+1}\left(t^{\prime}\right)\right]}\right\rangle .
$$

Now comes the crux of the matter: the assumption $T \ll W$, already utilized to allow a perturbative treatment in the leading order of $T$, also leads to a separation of timescales between the rate of change of the probabilities and the dephasing. This will be checked explicitly at the end of the calculation. Under this assumption, $P_{j}(t)$ can be taken out of the averaging. Thus $I=P_{j}(t) Q$, with $Q=\left\langle\int_{0}^{t} e^{i\left[\phi_{j}(t)-\phi_{j}\left(t^{\prime}\right)\right]} e^{i\left[\phi_{j+1}(t)-\phi_{j+1}\left(t^{\prime}\right)\right]}\right\rangle$.

Assuming $t \gg \tau_{\phi}$, we obtain that $Q$ is a constant, related to the dephasing correlation function $C_{\phi}(\Delta T)$ found before, through the relation $Q=\int_{0}^{\infty} C_{\phi}^{2}(t)$. 
Using this result Eq. (12) takes the form:

$$
\frac{d P_{j}}{d t}=2 T^{2} Q\left[P_{j+1}+P_{j-1}-2 P_{j}\right]
$$

where we have omitted the averaging notation. This is the well-known diffusion master equation. This implies the (ensemble-averaged) probabilities will indeed diffuse, with a diffusion coefficient $D=2 T^{2} Q$.

Combining this with Eq. (5) for the dephasing correlation function, we obtain our main result:

$$
D=2 T^{2} \int_{0}^{\infty} C_{\phi}^{2}\left(t^{\prime}\right) d t^{\prime}=2 T^{2} \int_{0}^{\infty} e^{-2 \int_{0}^{t^{\prime}}\left(t^{\prime}-t\right) C(t) d t} d t^{\prime}
$$

For the case of short correlation time $\tau W \ll 1$, we can take $C_{\phi}\left(t^{\prime}\right)$ from Eq. (6), and obtain:

$$
D=\frac{T^{2}}{\beta W^{2} \tau}=\frac{2 T^{2}}{\int_{-\infty}^{\infty} C(t) d t}
$$

This complies with the results of Ovchinnikov [1], who worked in the limit of $\tau \longrightarrow 0$ (delta-function correlations of the noise).

The main advantage of our method, other than the clear physical picture gained for the diffusion process, is that we obtain explicit analytical results also for the regime of long correlation times, as well as the crossover between the two regimes. For long correlation times $\tau W \gg 1$, Eq. (7) gives $C_{\phi}\left(t^{\prime}\right)$. Plugging this into Eq. (16) we obtain:

$$
D=\frac{\sqrt{2 \pi} T^{2}}{W}
$$

The crossover between the two regimes (short and long noise correlation times), is also described by Eq. (16). The results depend on the form of the noise correlation function $C(t)$. Notice that for $\tau W \sim 1$ Eqs. (17) and (18) give a result of the order of $T^{2} \tau$, which is where the two limits 'match'.

We have tested the theory numerically, on a finite lattice. The procedure is as follows:

1. We solve Eq. (10) numerically, starting with a probability distribution concentrated on a single site, and with a fluctuating disorder. For convenience, we take noise with linearly decaying correlations, i.e., $C(t)=$ $W^{2}(1-|t| / \tau) \theta(\tau-|t|)$, where $\theta$ is the Heaviside step function. The wavepacket spreads, as is illustrated in Fig. 1.

2. We ensemble-average the probability distribution over many realizations of the noisy environment. We find that the standard deviation of the ensemble-averaged probability distribution grows as $\sigma(t) \sim \sqrt{2 D t}$, as predicted by the theory. This is demonstrated in Fig. 2
From this dependence we extract the diffusion constant $D$. We find indeed that $D$ depends on the strength of the noise $W$ as well as its correlation time $\tau$.

Fig. 3 compares the result of the simulations with the theoretical prediction of Eq. (16), with a linearly decaying $C(t)$, as used in the simulations. Since the diffusion constant is proportional to $T^{2}$, we know from dimensional analysis that $D=T^{2} \tau f(W \tau)$, where $f(x)$ is de- (a)
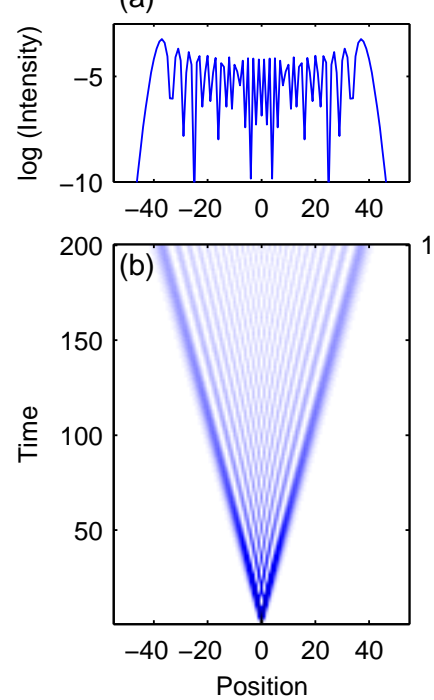

(c)
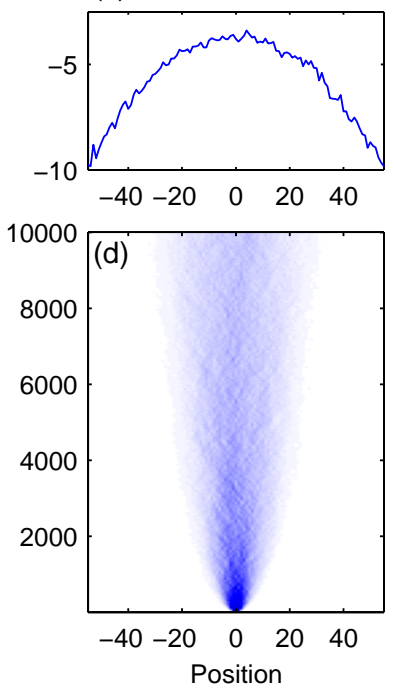

FIG. 1: The spread of a quantum-mechanical wavepacket in a perfectly periodic lattice $(T=1, W=0)$ showing ballistic spreading (a,b), versus a noisy lattice with $T=1, \tau=0.01$, $W=20$ showing diffusive spread $(\mathrm{c}, \mathrm{d})$. The wavefunction is confined to a single lattice site at $\mathrm{t}=0$. The probability distribution of the quantum particle after some propagation is plotted on a semilog scale in (a) and (c), showing a ballistic profile and a diffusive (gaussian) profile, correspondingly.

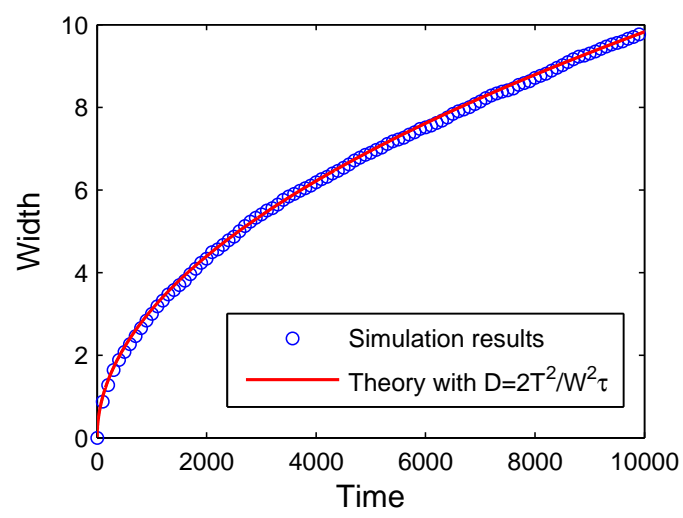

FIG. 2: A plot of the width versus time of a quantum mechanical wavefunction spreading in a noisy potential, showing diffusive propagation. The simulation parameters are $T=1$, $\tau=0.01, W=20$. The data is averaged over 100 realizations of the disordered potential. The numerical results (open circles) fit the theory (line), with no fitting parameters. 
termined from Eq. (16). This allows for a unified presentation of both regimes, which are manifested as different asymptotic regimes of the function $f(x)$ : for $W \tau \ll 1$ we know from Eq. (17) that $f(x) \sim 1 / x^{2}$ with a nonuniversal proportionality constant, while for $W \tau \gg 1$ we have $f(x)=\sqrt{2 \pi} / x$, as deduced from Eq. (18). Indeed, we numerically obtain scaling of this form, where the data for different runs collapse onto a single curve, when scaled correctly. Fig. 3 shows $f(x)$ derived numerically, and compares it to the prediction of Eq. (16). Note that there are no fitting parameters. The excellent fit confirms the validity of the analytical approach used.

Summary.- We have considered a tight-binding model with random on-site energies, fluctuating in time with an arbitrary correlation time. Using the separation of timescales between the (slow) diffusion process and the (fast) dephasing, we managed to map the complex evolution of the probabilities onto the problem of dephasing of a single site. For a noisy environment with short correlation time (compared to the reciprocal strength of the disorder, $1 / W)$, the phenomenon of motional narrowing is manifested in the diffusion constant. In the regime of long correlation time, we show that the diffusion constant is inversely proportional to the strength of the disorder.

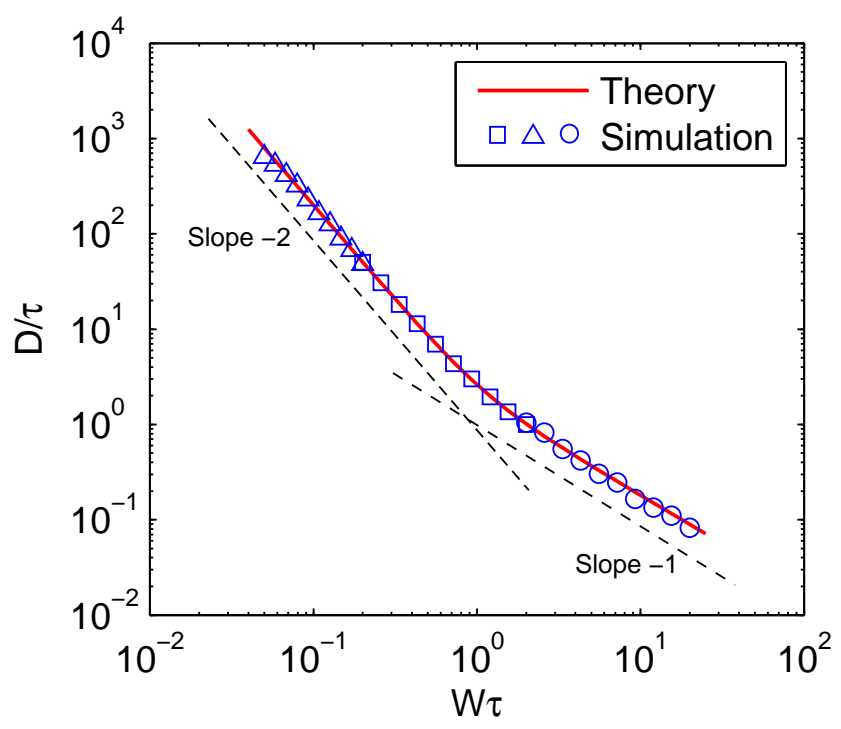

FIG. 3: A fit of the theoretical results to numerical calculations. The diffusion constant was found numerically, for correlation times $\tau=0.01$ (triangles), $\tau=0.1$ (squares) and $\tau=1$ (circles) and noise magnitudes ranging from $W=2$ to $W=20$. Each point in the graph is an average over 50 realizations. As expected theoretically, there is a crossover from a regime with slope -2 (i.e., $1 / x^{2}$ dependence), to a regime with slope -1 ( $1 / x$ dependence). The different curves overlap at points with the same value of $W \tau$. The form of the crossover is given by Eq. (16), integrated numerically for the intermediate regimes. No fitting parameters are used in the comparison.
We also give a solution for the crossover regime. Our results show excellent agreement with simulations, inadvertently confirming previous results obtained for the some particular cases studied in the past, but continuing much beyond to give a general solution for the full range of the parameter space. This includes quantum systems under more realistic noise environments conditions, which have not been studied before. Our results and exact solutions are applicable to various quantum systems, from diffusion of electrons and excitons to photon propagation in coupled waveguides, and to the general question of quantum random walks with decoherence.

We dedicate this work to the late Y. Levinson, who contributed to it by illuminating discussions, and pointed out the striking resemblance to the physics of motional narrowing. We would like to thank Y. Oreg and Y. Imry for useful discussions. A.A. acknowledges funding by the Israel Ministry of Science and Technology via the Eshkol scholarship program. Y.L. acknowledges support by the Israel Academy of Science and Humanities via the Adams scholarship program.

[1] A. Ovchinnikov and N. Erikhman, Sov. Phys. JETP 40, 733 (1974).

[2] A. Madhukar and W. Post, Phys. Rev. Lett. 39, 1424 (1977).

[3] A. M. Jayannavar and N. Kumar, Phys. Rev. Lett. 48, 553 (1982).

[4] D. Kumar, Phys. Rev. A. 29, 1571 (1984).

[5] J. Heinrichs, Z. Phys. B 57, 157 (1984).

[6] L. Golubovic, S. Feng, and F.-A. Zeng, Phys. Rev. Lett. 67, 2115 (1991).

[7] P. Sheng and Z.-Q. Zhang, Phys. Rev. B. 48, 12609 (1993).

[8] Y.-K. Yu, Phys. Rev. Lett. 85, 4199 (2000).

[9] V. Kendon, Mathematical Structures in Computer Science pp. 1169-1220 (2007).

[10] Y. Yin, D. E. Katsanos, and S. N. Evangelou, Phys. Rev. A 77, 022302 (2008), 0708.1137.

[11] C. Madigan and V. Bulovic, Phys. Rev. Lett. 96, 046404 (2006).

[12] H. B. Perets, Y. Lahini, F. Pozzi, M. Sorel, R. Morandotti, and Y. Silberberg, Phys. Rev. Lett. 100, 170506 (2008).

[13] Y. Silberberg, Y. Lahini, Y. Bromberg, E. Small, and R. Morandotti, arXiv (2008), 0812.0223.

[14] A. S. Pikovsky and D. L. Shepelyansky, Phy. Rev. Lett. 100, 094101 (2008).

[15] G. Kopidakis, S. Komineas, S. Flach, and S. Aubry, Phys. Rev. Lett. 100, 084103 (2008).

[16] S. Flach, D. O. Krimer, and C. Skokos, Phys. Rev. Lett. 102, 024101 (2009).

[17] S. Zhang, J. Park, V. Milner, and A. Z. Genack, Phys. Rev. Lett. 101, 183901 (2008).

[18] T. Holstein, Ann. Phys. 8, 343 (1959).

[19] M. Esposito and P. Gaspard, Phys. Rev. B 71, 214302 (2005). 
[20] Y. Dubi and M. D. Ventra, arXiv (2008), 0812.4774.

[21] P. W. Anderson, Phys. Rev. 109, 1492 (1958).

[22] S. Marianer and J. M. Deutsch, Phys. Rev. B. 31, 7478 (1985).

[23] K. Kitahara and J. Haus, Z. Physik B 32, 419423 (1979).

[24] G. D. Mahan, Many-particle Physics (Plenum, New York, 1981).
[25] O. Firstenberg, M. Shuker, A. Ben-Kish, D. R. Fredkin, N. Davidson, and A. Ron, Phys. Rev. A. 76, 013818 (2007).

[26] C. Slichter, Principles of Magnetic Resonance (Springer, Berlin, 1990). 